\title{
5. Zur Ethik menschlicher Embryoidmodelle: die Schaffung einer konsistenten gesellschaftlichen Vereinbarung
}

Übersetzt von Lilian Marx-Stölting und Angela Osterheider

Als Embryoide werden mehrzellige Gebilde bezeichnet, die natürlichen Embryonen sowohl in Bezug auf die enthaltenen Zelltypen als auch auf die dreidimensionale Organisation ähneln. In diesem Kapitel erörtern wir den ethischen und rechtlichen Umgang mit menschlichen Embryoidmodellen und argumentieren, dass die (bisherige) Einbettung dieser Frage in die Debatte um den moralischen Status des Embryos weder $\mathrm{zu}$ einem gesellschaftlichen Konsens noch zu einer umsetzbaren Politik führen wird. Wir zeigen, dass die Bestimmung unserer gesellschaftlichen Prioritäten entscheidend ist, um für diese neuen Entitäten eine konsistente ethische Richtlinie entwerfen zu können. Nach einem Überblick über die jüngsten Fortschritte auf diesem Forschungsgebiet unterscheiden wir ethische Fragen, die durch verschiedene Arten von Embryoiden aufgeworfen werden: (1) Embryoide, die (nur) Bestandteile von Embryonen nachbilden, und (2) Embryoide, die den Embryo als Ganzes nachbilden. Innerhalb der zweiten Kategorie diskutieren wir die Einordnung dieser Embryoide bezüglich der „14Tage-Regel“" (die in Ländern, die Forschung mit Embryonen zulassen, weit verbreitete Grenze für die Kultivierung natürlicher Embryonen in vitro bis zum 14. Tag nach der Befruchtung) und ziehen daraus Schlussfolgerungen für zukünftige Regelungen.

Dieses Kapitel versteht sich damit als Aufruf zu mehr Konsistenz in der biomedizinischen Forschung mit menschlichen Materialien; im Zuge dessen wird versucht, Embryoide innerhalb eines Spektrums bestehender Praktiken zu verorten, das von der Stammzellforschung oder künstlichen Befruchtung (IVF) bis hin zur Forschung an Menschen reicht. Die gegenwärtige Praxis, menschliche Embryonen ohne Rücksicht auf ihren potenziellen Nutzen einzufrieren oder zu verwerfen widerspricht der 
Annahme einer besonderen Berücksichtigung von menschlichem Material. Im Unterschied dazu wird mit der Schaffung synthetischer Modelle zu Forschungszwecken gerade anerkannt, dass kein menschliches Material unnötig und ohne Prüfung verwendet werden, sondern im Rahmen hochrangiger Forschung immer der Menschheit dienen sollte. Wir argumentieren, dass es an der Zeit ist, das vollständige Verbot der Embryonenforschung - bezogen auf natürliche oder synthetische Embryonen - über die zulässigen 14 Tage hinaus zu überdenken, und dass die Forschung an synthetischen Embryonen dabei kontextabhängig beurteilt werden sollte: Je weiter das Stadium der synthetischen Embryonen fortgeschritten ist, desto mehr wissenschaftliche Begründungen, gesellschaftliche Vorteile und Kontrollen sowie Beurteilungen des Nutzens, der Absichten, der Notwendigkeit, der Risiken und des Nutzens des jeweiligen Forschungsprojekts sind erforderlich.

\subsection{Einführung}

Wissenschaftliche Innovationen lösen oft Befürchtungen aus. Das trifft ganz besonders $\mathrm{zu}$, wenn es um menschliche Embryoide geht. In den letzten Jahren hat sich ein sich rasch entwickelndes Forschungsgebiet herausgebildet, das untersucht, wie man Embryonen aus ihren Grundbestandteilen - pluripotenten Stammzellen und deren Derivaten - herstellen kann (Shahbazi et al., 2019). Infolgedessen wurde in sensationsheischenden Schlagzeilen über die Herstellung "synthetischer Menschen“ in Laboratorien berichtet, während die alltägliche Realität der wissenschaftlichen Forschung in einem Labor weit von diesen eingängigen Schlagzeilen entfernt ist. Darüber hinaus hat es das politische Klima, zumindest in den USA, fast unmöglich gemacht, über den ethischen Status von Embryoiden zu diskutieren, ohne sofort die Tür zu einer breiteren Diskussion über Abtreibung zu öffnen. Die Heftigkeit dieser Debatte hat dabei zu einer verzerrten Darstellung dieser komplexen technologischen Innovationen beigetragen. Gleich der Bemühungen, sich auf technische Einzelheiten zu fokussieren, kehren wir immer wieder zu der umfangreichen und unlösbaren Debatte über die moralische Unantastbarkeit des Embryos zurück. Darüber hinaus haben die jüngsten Einschränkungen der Finanzierung durch die National Institutes of Health (NIH) in den USA für die Forschung an embryonalen Modellen und embryoähnlichen Strukturen dazu geführt, dass es für Wissenschaftler/-innen noch schwieriger geworden ist, sich Gehör zu verschaffen und klar zum Ausdruck zu bringen, dass es nie die Absicht und das langfristige Ziel ihrer Forschung war, Menschen in einem Labor zu erzeugen. Folglich besteht ein stillschweigender Konsens, sich eher bedeckt zu halten, während man sich nach einem günstigeren sozio-politischen Klima sehnt, und trotz 
zahlreicher Aufrufe zur Aufnahme einer internationalen Diskussion wurden bisher nur sehr wenige Neuregelungen vorgeschlagen. Wir schlagen dagegen vor, die Debatte von der Diskussion über den moralischen Status des Embryos zu verlagern auf die Definition einer gesellschaftlichen Vereinbarung, das der klinischen Forschung zugute käme und gleichzeitig anerkennen würde, dass kein menschliches Material leichtfertig ohne angemessene ethische Kontrolle verwendet werden sollte.

\subsection{Aktueller Stand der Forschung an menschlichen Embryoiden}

Die Gewinnung von embryonalen Stammzellen des Menschen und der Maus hat die Möglichkeit eröffnet, mit embryonalen Bestandteilen zu arbeiten. Eine spannende Herausforderung ist dabei der Versuch, die minimal notwendigen physikalischen und chemischen Bedingungen zu erkunden, die ihre Selbstorganisation in Embryoide auslösen können, vielzellige Einheiten, die natürlichen Embryonen ähneln, nicht nur hinsichtlich der Zelltypen, sondern auch der 3-D-Organisation. Dieser Bottom-up-Ansatz in der Embryologie, Embryonen aus ihren Basiskomponenten wiederaufzubauen, hat das Potenzial, die grundlegenden Prinzipien der Säugetierembryogenese aufzudecken, was Auswirkungen auf die Reproduktions- und Regenerationsmedizin haben dürfte.

Bei der Entwicklung der Maus und des Menschen werden ähnliche Zwischenstadien durchlaufen, allerdings mit zeitlicher Verzögerung und morphologischen Unterschieden (siehe Abbildung 1).

Kurz nach der Befruchtung teilt sich die einzellige Eizelle und wird zu einem Ball von Zellen, die sich verdichten und bald darauf in die drei Zelltypen unterteilen, die später die Blastozyste bilden. Während sich jeder dieser Zelltypen rasch ausdehnt, kommt es zum wichtigsten Schritt in der Entwicklung: zur Gastrulation. In diesem Stadium bildet sich eine vorübergehende Struktur, der sogenannte Primitivstreifen, der die radiale Symmetrie (Kugelsymmetrie) der embryonalen Scheibe (Epiblast) aufbricht und zum Entstehen der drei embryonalen Keimschichtderivate führt: Ektoderm, Mesoderm und Entoderm, die die Grundlage für den Bauplan des Körpers und die Ausrichtung der embryonalen Achsen bilden. Die zellulären Nachkommen der embryonalen Keimschichten bringen alle Organe des erwachsenen Körpers hervor (siehe Einleitung, Kap. 2.1). Aus dem Ektoderm entsteht z. B. das gesamte Nervensystem, die Sinnesorgane und die Haut; aus dem Mesoderm das Blut, das Herz, die Muskeln, die Knochen und aus dem Entoderm u. a. die Lunge, der Darm, die Bauchspeicheldrüse und die Leber. Die anschließende Morphogenese der drei Keimschichten liefert die Anlagen für die verschiedenen Organe, die weiterwachsen, um schließlich reif und funktionsfähig 
Abbildung 1: Zeitleiste der frühen Entwicklung von Mensch und Maus

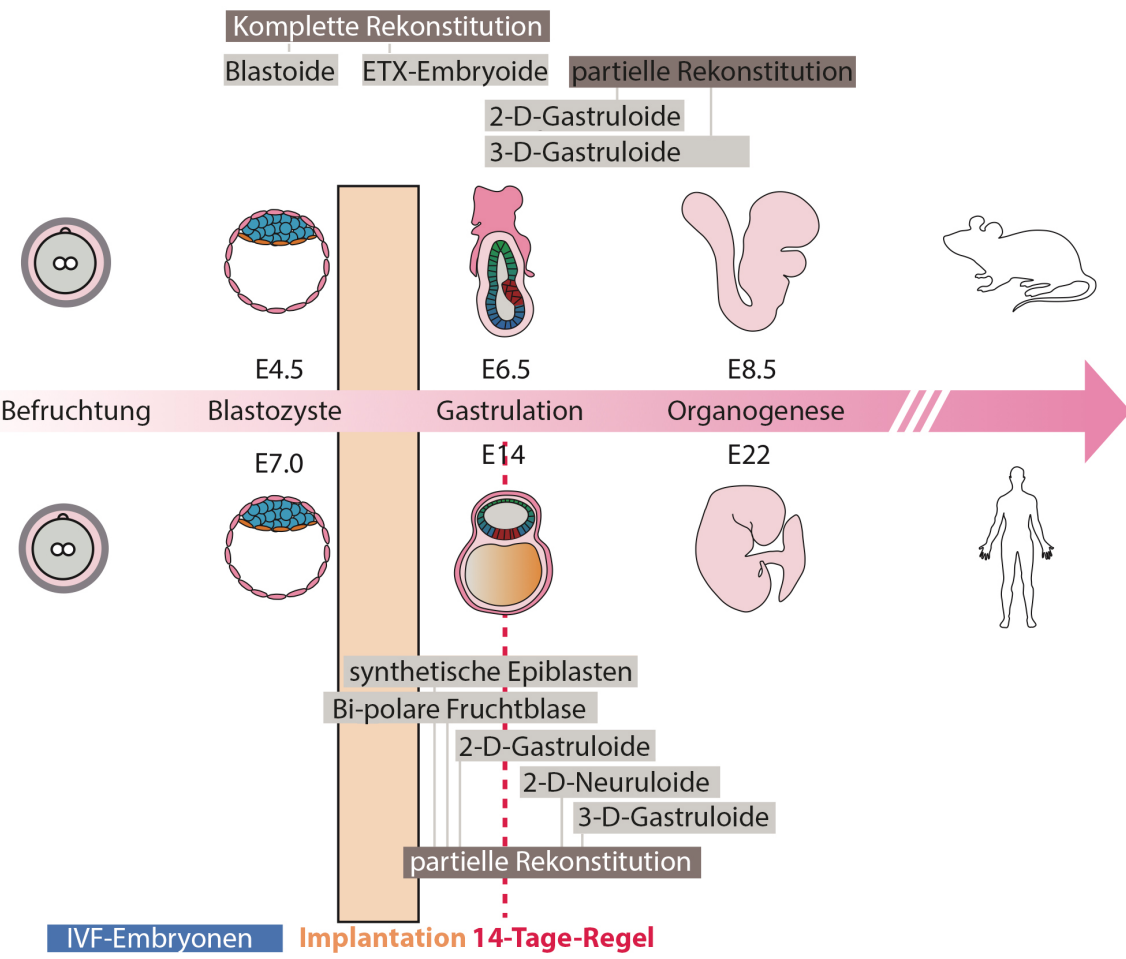

zu werden (siehe Abbildung 1). Embryonalzellen, die sich außerhalb des Epiblasten befinden, erzeugen extra-embryonales Gewebe, das für die Anheftung des Embryos an die Gebärmutter und die Bildung von Plazenta und Nabelschnur notwendig ist.

Aus dieser kurzen Beschreibung wird deutlich, dass das Vorhaben, einen Embryo von Grund auf neu aufzubauen, eine schwierige Aufgabe ist. Zum einen besteht eine offensichtliche architektonische Herausforderung, da Embryonen in jedem Stadium aus mehreren Zelltypen bestehen, die in einer eigentümlichen Architektur angeordnet sind. Zum anderen - und sogar noch herausfordernder - ist die frühe Embryonalentwicklung sehr dynamisch, und die Formveränderungen, die in den ersten Wochen der Entwicklung auftreten, sind die entscheidendsten, die in der Lebensspanne eines Organismus auftreten. Trotz dieser Hindernisse gehen wir davon aus, dass es technisch möglich werden könnte, Embryoide zu erzeugen, die mithilfe bestimmter biotechnolo- 
gischer Methoden hergestellt werden und in der Lage sind, sich in spätere Stadien weiterzuentwickeln.

Innerhalb dieses Rahmens hat sich die synthetische Embryologie im letzten Jahrzehnt etabliert (siehe auch Shahbazi et al., 2019). Da die Maus aus historischer Perspektive das Modell der Wahl für die Embryologie von Säugetieren war, überrascht es nicht, dass Mausembryoide derzeit deutlich weiter entwickelt sind als menschliche Embryoide. Es können zwei Arten von Embryoiden unterschieden werden: diejenigen, die auf eine (möglichst) vollständige Rekonstitution eines bestimmten Embryonalstadiums abzielen, wobei alle Zelltypen in der spezifischen Geometrie vorliegen, und diejenigen, die eine teilweise (partielle) Rekonstitution eines Embryos mit nur einer Teilmenge der in einem bestimmten Embryonalstadium vorhandenen Zelltypen darstellen. Das Konzept, einen nahezu (d. h. soweit dies in einem In-vitro-Modell möglich ist) vollständigen Embryo aus pluripotenten embryonalen Zellen nachbilden zu können, ist ein Erbe der klassischen experimentellen Embryologiestudien des Amphibiensystems, die letztlich zum Klonen des ersten Tieres, eines Frosches (Gurdon, 1962), führten. Bisher haben nur wenige Studien zu Säugetiersystemen über die Erzeugung von fast „vollständig rekonstituierten“ frühen Embryonen im Blastozystenstadium („Blastoide“; Rivron et al., 2018a; Li et al., 2019; Sozen et al., 2019) oder Gastrulationsstadium („Gastruloide“; hier auch „ETX-Embryoide“"1; Sozen et al., 2018) berichtet, und dies auch nur bei der Maus (siehe Abbildung 1). Diese Studien nutzen die Möglichkeit, aus embryonalen Stammzellen der Maus unabhängige und stabile Kulturen der verschiedenen Zelltypen herzustellen, die die Blastozyste bilden. Dies hat die Herstellung von Embryoiden durch einen Ansatz erleichtert, bei dem die verschiedenen Zelltypen unabhängig voneinander hergestellt und unter bestimmten Bedingungen zusammengebracht werden. Bemerkenswert ist, dass die verschiedenen Zelltypen, sobald sie einmal vorliegen, in der Lage sind, sich mit unterschiedlicher Effizienz selbst $\mathrm{zu}$ embryonalen Anordnungen zusammenzufügen. Bis jetzt sind dies die einzigen erfolgreichen Versuche, fast vollständige Mausembryoide zu erzeugen, die möglicherweise eingepflanzt werden könnten, um eine Schwangerschaft bei Mäusen einzuleiten. Bislang haben sowohl „Blastoide“ als auch „ETX-Embryoide“ beim Transfer in vivo den Prozess der Einnistung in die Gebärmutter (Implantation) eingeleitet, was auch die weitere Morphogenese des Embryoids auslöste; aber die so entstandenen Strukturen konnten aus unbekannten Gründen in vivo keine späteren Entwicklungsstadien erreichen.

1 ETX steht für die Anfangsbuchstaben der drei verwendeten Maus-Zelllinien. 
Drei weitere populäre Methoden haben eine „partielle Rekonstitution“ des menschlichen oder Mausembryos beginnend bei den Gastrulationsstadien erreicht:

a) Bei 2-D-Gastruloiden wurden Mikrostrukturierungstechniken eingesetzt, um pluripotente Stammzellen in Kolonien definierter Form und Größe zu erhalten (Warmflash et al., 2014). In dieser Versuchsanordnung ist die geometrische Eingrenzung der Kolonien ausreichend, um dynamische Signalwellen zu erzeugen, die die radiale Selbstorganisation der Keimschichten induzieren (Chhabra et al., 2019). Während in diesem System extra-embryonale Bestandteile vorkommen, ist die Geometrie zweidimensional und die dreidimensionale Struktur der Gastrula wird nicht nachgebildet. Eine Erweiterung dieser Methode hat „Neuruloide“ hervorgebracht, die eine sehr genaue Nachbildung des ektodermalen Bereichs in den Neurulationsstadien nach der Gastrulation darstellen. Sie verfügen über alle der vier wichtigsten ektodermalen Gewebe: Nervengewebe, Neuralleiste, sensorische Plakode (aus der sich später etwa die Linse des Auges oder die Sinneszellen des Innenohrs entwickeln) und Haut, die ähnlich wie beim natürlichen Embryo nebeneinander liegen (Haremaki et al., 2019). Diese selbstorganisierenden synthetischen Modelle, die man zu Tausenden herstellen kann, sind wertvolle Werkzeuge für aktuell forschende Embryologinnen und Embryologen, da sie eine unbegrenzte Quelle biologischen Materials bieten, um Stadien unserer eigenen Entwicklung zu erforschen, die sonst nicht untersucht werden könnten.

b) Der zweite Embryoid-Typ konzentriert sich ausschließlich auf den Kern des Embryos: den Epiblasten. ${ }^{2}$ Bei diesem Ansatz wird er als eine Kugel, ein synthetischer Epiblast, dreidimensional modelliert und kann dann durch den Einsatz von Morphoge$n^{3}{ }^{3}$ zur Gastrulation veranlasst werden (Shao et al., 2017; Simunovic et al., 2018). Es handelt sich dabei um eine partielle Darstellung eines Embryos, da Nervengewebe ebenso wie alle extra-embryonalen Zelllinien vollständig fehlen.

c) Schließlich beruhen 3-D-Gastruloide auf pluripotenten Stammzellaggregaten, die durch einen Impuls eines Wachstumsfaktors zur Differenzierung angeregt werden

2 Der Säugetier-Embryo (einschließlich des Menschen) enthält im Blastozystenstadium verschiedene Bereiche, die später unterschiedliche Funktionen haben. Aus dem Embryoblasten gehen später alle Gewebe des eigentlichen Embryos hervor. Der Trophoblast bildet die äußere Begrenzung der Blastozyste, ist für die Ernährung zuständig und bildet den embryonalen Teil der Plazenta. Als Epiblast bezeichnet man einen Teil des Embryoblasten, der dem Trophoblasten anliegt und den äußeren Teil der Keimscheibe bildet. Er besteht aus speziellem Epithel und bildet später den Primitivstreifen, der den Beginn der Gastrulation markiert, während der die drei Keimschichten Entoderm, Mesoderm und Ektoderm entstehen.

3 Morphogene sind Signalmoleküle, die die Entstehung von Mustern und Formen während der Entwicklung von Vielzellern steuern. Werden sie dem Kulturmedium beigefügt, lösen sie in vitro verschiedene Differenzierungsschritte aus. 
und sowohl aus Mauszellen (Beccari et al., 2018) als auch aus menschlichen Zellen (Moris et al., 2020) gewonnen wurden. 3-D-Gastruloide bieten eine axiale Organisation, die an die anterio-posteriore embryonale Achse (von vorne bis hinten) erinnert. Sie liefern eindrucksvolle Modelle der mesodermalen Entwicklung mit Zellbewegungen, die zur Verlängerung der embryonalen Achse und zur Bildung von Muskeln (Somitogenese) (Moris et al., 2020) führen. Wie synthetische Epiblasten sind sie eine partielle Darstellung eines Teils des Embryos, dem neurales Gewebe und extra-embryonale Bestandteile fehlen.

Während in einigen Schlagzeilen über die Erzeugung lebensfähiger synthetischer Embryonen, die sich zu einem Menschen entwickeln können, berichtet wird, ist die Realität der angesprochenen Methoden zumindest im Moment noch weit hiervon entfernt. Erstens konzentrierten sich die meisten Arbeiten in diesem Bereich auf eine nur teilweise Rekonstitution embryonaler Teile. Sie sind daher per definitionem nicht in der Lage, sich zu einem Menschen zu entwickeln. Wie oben bereits erwähnt, ist nur für die Maus über vollständig rekonstituierte Embryoide berichtet worden, und zwar entweder im Blastozystenstadium vor der Implantation oder im frühen Gastrulationsstadium, etwa zum Zeitpunkt der Einnistung. Darüber hinaus konnten sich diese Embryoide nach der Einpflanzung in die Gebärmutter der Maus zwar etwas weiter entwickeln und die Einnistung einleiten, sie sind aber bei Weitem nicht in der Lage, sich bis zur Geburt zu entwickeln, da ihre Entwicklung in vivo sehr früh endet. Die Anpassung der bei Mäusen verwendeten Herstellungsverfahren zur Bildung von menschlichen Blastoiden oder ETX-Embryoiden ist zweifellos nur eine Frage der Zeit. Um Protokolle, die für die vollständige Rekonstitution von Embryonen in der Maus entwickelt wurden, an die menschliche Umgebung anzupassen, sind Methoden zur Gewinnung menschlicher extra-embryonaler Zellen erforderlich. Diese werden derzeit unter Hochdruck entwickelt (Gao et al., 2019; Dong et al., 2020). Sobald diese menschlichen Embryoide erzeugt worden sind, wäre es theoretisch möglich, diese in eine menschliche Gebärmutter einzupflanzen. Obwohl vom jetzigen Stand der Technik bis zur Erzeugung entwicklungsfähiger menschlicher Embryonen noch ein langer Weg zurückzulegen ist, könnte dies vor dem Hintergrund der in den letzten Jahren erzielten Fortschritte innerhalb des nächsten Jahrzehnts geschehen.

\subsection{Der aktuelle Stand der Regulierung menschlicher Embryonenmodelle}

Gegenwärtig gibt es wenige explizite Vorschriften $\mathrm{zu}$ in vitro selbstorganisierenden menschlichen Embryonenmodellen, die aus pluripotenten Zellen abgeleitet und in 
räumlich begrenzter Anordnung kultiviert wurden (Matthews/Yang, 2018). Es ist unklar, inwieweit die 14-Tage-Regel - die weithin (z. B. in den USA und Großbritannien) angewandte Grenze für die Kultivierung natürlicher Embryonen in vitro bis zu 14 Tage nach der Befruchtung (Department of Health \& Social Security, 1984; Ethics Advisory Board, 1979) - auf menschliche Embryonenmodelle übertragen werden kann. Darüber hinaus ist unklar, wie diese Regel auf Embryoide übertragen werden kann, von denen bekannt ist, dass sie eine zeitlich veränderte Entwicklung zeigen und so die Zeitachse der Entwicklung normaler Embryonen umgehen können.

Die aktuell gültige Richtlinie der Internationalen Gesellschaft für Stammzellforschung (ISSCR) aus dem Jahr 2016 enthält wenige Vorschläge zur Regelung embryoähnlicher Strukturen: Bei „Forschung, welche die In-vitro-Kultivierung von Embryonen oder die experimentelle Erzeugung embryoähnlicher Strukturen, die sich zu einem menschlichen Organismus entwickeln können, beinhaltet“, sollen, nach Überprüfung durch einen Prozess zur Beaufsichtigung der Forschung an menschlichen Embryonen (genannt EMRO, engl. Human Embryo Research Oversight) als zulässig erachtet werden, solange „möglichst geringe Zeiträume für die In-vitro-Kultivierung gemäß einer stringenten wissenschaftlichen Grundlage sichergestellt werden" ${ }^{4}$ (ISSCR, 2016: 9). ${ }^{5}$

Nach der ISSCR sollten die beiden folgenden Forschungsaktivitäten verboten werden:

a) Das „In-vitro-Kultivieren eines intakten menschlichen Präimplantationsembryos oder einer organisierten embryoähnlichen Zellstruktur, die sich zu einem menschlichen Organismus entwickeln kann, ungeachtet des Verfahrens zu ihrer Gewinnung, ab Tag 14 oder nach Bildung des Primitivstreifens, je nachdem was früher eintritt" (ebd.).

b) „Experimente, bei denen eine Gestation menschlicher Embryonen oder organisierter embryoähnlicher Zellstrukturen, die sich zu einem menschlichen Organismus entwickeln können, ex utero oder in einem nicht-menschlichen Tieruterus erfolgt“ (ebd.).

4 Offizielle deutsche Übersetzung unter: https://www.isscr.org/docs/default-source/all-isscr-guide lines/guidelines-2016/isscr_germanguidelines_updates_03.pdf?sfvrsn=2 [11.08.2020].

5 Die Fähigkeit, einen Organismus zu bilden, wird auch als organismisches Potenzial bezeichnet. Paradoxerweise kann man erst sicher wissen, ob ein Embryoid organismisches Potenzial hat, wenn man es sich bis hin zu einem Organismus entwickeln lässt - und das heißt bis zur Geburt, was jedoch aus ethischen Gründen weltweit abgelehnt wird (Verbot des reproduktiven Klonens). 
Diese Leitlinien ermöglichen die Embryonenforschung vor 14 Tagen, stehen aber im Gegensatz zu den jüngsten Einschränkungen der NIH über die Finanzierung der Forschung an jeder Art von Embryoiden in allen Stadien; wodurch die Debatte geschürt wird. Vor dem Hintergrund, dass die ISSCR-Richtlinie Forschungsaktivitäten an synthetischen Embryonen nach 14 Tagen verbietet, argumentieren wir hier, dass es an der Zeit ist, das vollständige Verbot der Embryonenforschung - für natürliche und synthetische Embryonen - über die 14-Tage-Regel hinaus zu überdenken.

\subsection{Zwei Sackgassen in der Debatte}

Im Allgemeinen wurde die Debatte häufig wie folgt dargestellt: Sollten menschliche Embryoide den gleichen ethischen Status haben und als solche mit derselben ethischen Beaufsichtigung der Forschung reguliert werden wie intakte menschliche Embryonen? Eine solche Rahmung setzt voraus, dass wir wissen, 1) was der moralische Status eines natürlichen Embryos ist und 2) ob synthetische Embryonen mehr als ein Modell sind und in die gleiche Kategorie wie natürliche Embryonen fallen sollten, $d . h$. ob sie ein Potenzial haben, sich zu einem Menschen zu entwickeln. Es ist allerdings unwahrscheinlich, dass diese Fragen beantwortet werden aus den folgenden Gründen:

\subsubsection{Das Fehlen eines Konsenses über den moralischen Status des Embryos}

Die Debatte über den moralischen Status eines natürlichen Embryos war von Beginn an ein Minenfeld und umfasst ein breites Spektrum von Positionen (Iltis et al., 2019). Manche sind der Ansicht, dass das Konzept des moralischen Status binär ist: Eine Entität hat entweder einen vollen moralischen Status mit einer Reihe von Rechten (George/Gómez-Lobo, 2005) oder sie hat keinen moralischen Status. Im Gegensatz dazu sehen andere den moralischen Status als entwicklungsbezogen emergent an, gehen also davon aus, dass er durch bestimmte Entwicklungsprozesse erst begründet ist und mit der Entwicklung ansteigt (Singer/Kuhse, 1986; De Grazia, 2008). Von der Ansicht, dass Embryonen von der Empfängnis an einen vollen moralischen Status haben, über die Ansicht, dass sie im Zuge ihrer Entwicklung einen höheren moralischen Status erlangen, bis hin zu der, dass sie überhaupt keinen moralischen Status haben, kann in der Öffentlichkeit oder auch in religiösen Gruppen kein Konsens erreicht werden. Meinungsverschiedenheiten über den moralischen Status eines Embryos bestehen sogar unter katholischen Gläubigen innerhalb der strengen Grenzen der römischen Kirchenorthodoxie (Congregation for the Doctrine of the Faith, 1987). Laut Aquin ist die Tötung erst dann Mord, wenn der Fötus von der rationalen Seele belebt ist (Ford, 
1989). Daher lassen manche Theologinnen und Theologen trotz des gegenwärtigen katholischen Konsenses, dass das menschliche Leben mit der Empfängnis beginnt, die Möglichkeit offen, den moralischen Status als biologisch emergent zu betrachten. Die Ansichten über den moralischen Status des Embryos beruhen inhärent auf unvereinbaren Werten der verschiedenen Parteien.

Eine eindrucksvolle Demonstration des fehlenden Konsenses ist, dass keine der beiden oben genannten etablierten Richtlinien, weder die 14-Tage-Regel selbst noch die Empfehlungen der ISSCR, auf dem moralischen Status basiert. Die 14-Tage-Regel ist nicht das Ergebnis eines Konsenses über den moralischen Status des menschlichen Embryos, sondern das Produkt einer vernünftigen Übereinkunft zwischen Parteien mit gegensätzlichen Ansichten. Sie hatte gerade den Vorteil, der Kultivierung des natürlichen Embryos eine klare und handhabbare Entwicklungsgrenze zu setzen, ohne sich dabei auf moralische Werte zu stützen. Doch trotz dieses fehlenden Konsenses in einer pluralistischen Gesellschaft befriedigen die jüngsten Beschränkungen der NIH-Finanzierung für die Forschung an embryonalen Modellen und embryoähnlichen Strukturen nur den Teil der Öffentlichkeit, der sich am stärksten gegen jegliche Forschung an Embryonen, ob natürlich oder synthetisch, ausspricht.

\subsubsection{Das Fehlen eines Konsenses bezüglich des Modellproblems}

Die andere besonders umstrittene Frage, ist, ob Embryoide mehr als ein Modell sind und ihnen folglich derselbe ethische Status zukommt wie intakten natürlichen Embryonen. Diese Frage kann durch einen ausführlichen Vergleich der Eigenschaften synthetischer und natürlicher Embryonen beantwortet werden. Dies kann auf vielen quantitativen Ebenen erfolgen, von der molekularen Signatur der verschiedenen Zelltypen über die Form der Gewebe bis hin zur gesamten Morphologie. Da die In-vitro-Strukturen jedoch immer komplexer werden und ihre analytische Beschreibung immer detaillierter wird, wird es immer schwieriger, eine Grenze zu ziehen und zu wissen, wann Ähnlichkeit zu einer Identität in der Natur wird. Wissenschaftler/-innen (Aach et al., 2017; Hyun et al., 2020) haben betont, dass die Modellfrage einen Teufelskreis darstellt: Modelle menschlicher Embryonen sollen Experimente an menschlichen Embryonen vermeiden, aber eigentlich wäre mehr Forschung an menschlichen Embryonen nötig, um zu wissen, ob synthetische menschliche Embryonen nur ein Paradigma oder mehr als ein Modell sind. Wir brauchen jetzt ein In-vivo-Äquivalent zu unseren Modell-Embryonen, um beurteilen zu können, welchem Entwicklungsstand die Modelle wie genau entsprechen. Unter der Annahme, dass es uns gelingen würde, diese Embryoide über den 14. Tag hinaus zu kultivieren, wäre es ohne diese Kontrollen 
sehr schwierig zu bestimmen, wie gut die In-vitro-Modelle natürliche embryologische Ereignisse, die in diesem Stadium auftreten, getreu wiedergeben. Der endgültige Beweis der Einnistung und Entwicklung eines synthetischen Embryos in einer natürlichen Gebärmutter kommt aus ethischen Gründen nicht in Frage. Selbst wenn dies technisch möglich wäre, was wahrscheinlich nur eine Frage der Zeit ist, sollten Embryonenmodelle nicht für die direkte Verwendung in der assistierten Reproduktion mit dem Ziel einer Schwangerschaft hergestellt werden.

\subsection{Entwicklung einer konsistenten gesellschaftlichen Vereinbarung}

Wie bereits erwähnt, ist es unrealistisch, irgendeine Art von Konsens über den moralischen Status des Embryos - natürlich oder synthetisch - zu erwarten, da diese Frage ohne eine philosophische Rahmung, die weit über die strikten Grenzen der Biologie hinausgeht, nicht beantwortet werden kann. Dass die Konfliktparteien in ihrer Argumentation nicht die gleichen Prämissen teilen, hat die öffentliche Debatte über den moralischen Status des Embryos in eine Sackgasse geführt, die den Gesetzgebern nicht dabei helfen wird, irgendeine Art der zukünftigen Regulierung zu entwerfen. Dies bedeutet keinesfalls, dass die Frage nach dem moralischen Status des menschlichen Embryos als solche gesellschaftlich irrelevant ist. Im Gegenteil, jede Partei vertritt Positionen, die zur Ausgewogenheit beitragen, indem sie die Art und Weise mit beeinflussen, wie wir das Thema einordnen. Diese dynamischen Spannungen erinnern uns daran, dass wir äußerste Vorsicht und Wachsamkeit walten lassen müssen, wenn es um die Forschung mit menschlichem Material geht und darum, den Raum für eine pluralistische Diskussion zu wahren. Da die Frage nach dem moralischen Status des Embryos bislang jedoch unzureichend war, um solide Regelungen zu liefern, ist es vielleicht an der Zeit, ihre Rahmung zu ändern. Die Frage „Was halte ich für den moralischen Status eines Embryos?" unterscheidet sich deutlich von der Frage „Was sollen wir als Gesellschaft in Bezug auf Embryonen tun?“. Sie ist auch ganz anders gelagert als die Frage hinsichtlich synthetischer Embryonen. Die Definition unserer gesellschaftlichen Prioritäten ist daher entscheidend, um für diese neuen Entitäten eine konsistente ethische Richtlinie entwerfen zu können (Annas et al., 1996).

\subsubsection{Der Fall der partiellen embryonalen Rekonstitution}

Der gegenwärtige Stand der Technik bietet nur eine teilweise und unvollständige Rekonstitution des intakten menschlichen Embryos. Dazu gehören Forschungsarbeiten, 
die nicht alle Zelllinien miteinbeziehen (z. B. synthetische Epiblasten, 3-D-Gastruloide, Strukturen der Fruchtblase) oder Modelle umfassen, bei denen wesentliche Merkmale und die 3-D-Architektur des Embryos eindeutig fehlen (z. B. 2-D-Gastruloide). Daher sind diese Embryoide nicht gleichwertig mit intakten Embryonen, da sie kein organismisches Potenzial besitzen, d. h. sich nicht bis zur Geburt entwickeln können. Als solche werfen sie keine ethischen Probleme auf und sollten nicht verpflichtend überprüft werden müssen. Schließlich könnten potenzielle ethische Fragen aufgeworfen werden, wenn diese Strukturen über lange Zeiträume kultiviert werden, um funktionelle Organe zu modellieren. Zerebrale Organoide und die Frage nach deren Bewusstseinszustand ist hierfür ein Beispiel (siehe zu dieser Frage auch Schicktanz, Kap. 6). Eine öffentliche Debatte über die moralischen Eigenschaften, die unsere Gesellschaft für wichtig erachtet, könnte zur Regulierung dieser Organoide genutzt werden, aber das würde den Rahmen dieses Kapitels sprengen und wird an anderer Stelle diskutiert (Aach et al., 2017).

\subsubsection{Ethische Überlegungen zur vollständigen embryonalen Rekonstitution vor der „14-Tage-Grenze“}

Wie bereits erwähnt, gibt es derzeit keinen Bericht über vollständige menschliche Embryoide, die ein Implantationspotenzial aufweisen könnten. Allerdings sollten wir auch zukünftige moralische Bedenken antizipieren. Einige werden vielleicht über die ethische Zulässigkeit von vollständigen synthetischen Modellen ausschließlich für Forschungszwecke diskutieren. Wir fordern an dieser Stelle jedoch ethische Konsistenz. Derzeit existieren über eine Million kryokonservierter ${ }^{6}$ Embryonen in IVF-Zentren und Kryobanken. 90.000 davon werden höchstwahrscheinlich jedes Jahr verworfen (Gurmankin et al., 2003; Gleicher/Caplan, 2018). Diese Zahlen sind so hoch, dass es nur eine Frage der Zeit ist, bis die stete Aufbewahrung von Embryonen für Kryobanken unerschwinglich wird. „Diese Embryonen werden derzeit entweder als bloßer Abfall behandelt oder bis zu ihrer unvermeidlichen Vernichtung kryokonserviert gehalten“ und werden daher nie für Forschungszwecke verwendet, sondern eher ,aufgegeben“ (Gleicher/Caplan, 2018: 141). Folglich haben Wissenschaftler/-innen die Einrichtung gemeinnütziger Biobanken für menschliche Embryonen zur Verwendung in der Forschung vorgeschlagen, da „ein höherer Zweck, eine stellvertretende Zustimmung und eine angemessene öffentliche Aufsicht in der Tat eine weitaus respektvollere Verwendung von aufgegebenen menschlichen Embryonen darstellen würden“ (ebd.). In eine

6 D. h. z. B. für eine spätere Nutzung eingefrorene Embryonen. 
ähnliche Richtung argumentieren auch Philosophen wie Sissela Bok (1988), Thomas Douglas und Julian Savulescu (2009) oder Michael Sandel (2005): Auf natürliche Weise gehen so viele Embryonen während der ersten Phase der Schwangerschaft verloren, dass, wenn wir ernsthaft über den Embryonenverlust besorgt wären, es notwendig wäre, einen monumentalen Kampf gegen alle Fehlgeburten zu führen. Wir befinden uns daher letztlich in einer paradoxen Situation, in der wir uns intensiv mit der ethischen Zulässigkeit synthetischer Embryonen befasst haben, während unsere Gesellschaft die große Zahl verworfener eingefrorener Embryonen aus der IVF oder auch durch frühe Fehlgeburten nicht in derselben Weise für ethisch bedenkenswert hält. Die Forschung mit Embryoiden hat jedoch das Potenzial, Behandlungen von Unfruchtbarkeit zu erforschen, und daher kann derartige Forschung langfristig zu einer geringeren Anzahl von Embryonen führen, die für einen erfolgreichen IVF-Zyklus benötigt werden. So kann die Gesamtzahl der verworfenen Embryonen verringert und können Fehlgeburten vermieden werden. Mit anderen Worten: Die Herstellung synthetischer Modelle für Forschungszwecke ist ethisch nicht problematischer als die Ansammlung eingefrorener Embryonen ohne jeglichen Nutzen für unsere Gesellschaft. Die gegenwärtige Praxis, menschliche Embryonen einzufrieren oder zu entsorgen ohne Beachtung ihres potenziellen Nutzens, widerspricht genau genommen der Annahme einer besonderen Berücksichtigung von menschlichem Material. Umgekehrt wird mit der Herstellung synthetischer Modelle für Forschungszwecke gerade anerkannt, dass kein menschliches Material ohne Grund und Prüfung verwendet werden, sondern bei der Beantwortung hochrangiger Forschungsfragen immer der Menschheit dienen sollte.

\subsubsection{Ethische Standards für die zukünftige Forschung an vollständigen Embryoiden nach 14 Tagen}

Wir müssen uns mit künftigen ethischen Bedenken befassen, die durch vollständige Embryoide aufgeworfen werden, die eines Tages die menschliche Embryonalentwicklung über 14 Tage hinaus nachahmen könnten. Für diese Entwicklungen ist höchstwahrscheinlich der Einsatz einer künstlichen Membran notwendig, um eine Einnistung in die Gebärmutterwand nachahmen zu können. Die Debatten hierzu sind besonders kontrovers, da es keinen Konsens über den moralischen Status des Embryos weder in diesem noch in späteren Stadien gibt.

Nehmen wir einmal an, dass ein 14-tägiger Embryo bereits den vollen moralischen Status hat und daher den gleichen Schutz verdient wie eine Person. Das bedeutet nicht notwendigerweise, dass jegliche Experimente oder Forschungen unbedingt ver- 
boten werden sollten. Die Forschung an Menschen zeigt, dass die moralische Würde einer Person an sich nicht jede Art von Forschung oder Experimenten ausschließt. Sie erfordert allerdings einen strengen und kohärenten Rahmen für die Bewertung der ethischen Zulässigkeit solcher Forschungsstudien. Unsere Gesellschaft hat hierfür z. B. die informierte Zustimmung zusammen mit anderen Prinzipien wie Transparenz, Verhältnismäßigkeit der Risiken und potenzieller Nutzen als Voraussetzung für Experimente an Menschen definiert.

Die ISSCR-Richtlinie aus dem Jahr 2016 stützt das Verbot der Kultivierung von Embryoiden nach 14 Tagen darauf, dass „ein breiter internationaler Konsens besteht, dass derartige Experimente einer stringenten wissenschaftlichen Grundlage entbehren, grundsätzliche ethische Fragen aufwerfen und/oder vielerorts illegal sind." (ISSCR, 2016: 9).

Wir argumentieren, dass wir diese Debatte wieder eröffnen sollten, da 1) die 14-Tage-Regel keine moralische Begründung hat. Viele Forschende haben diskutiert, ob das Auftauchen des Primitivstreifens als Ende der möglichen Entstehung von Zwillingen ein solides oder eher willkürliches Kriterium ist. Der Konsens besteht im Grunde nicht so sehr über den Inhalt einer solchen 14-Tage-Regel als vielmehr über die Erkenntnis, dass es für uns von Vorteil ist, eine Regel zu haben, auch wenn eine solche Regel willkürlich ist. 2) Wir sehen jetzt mehr und mehr den potenziellen medizinischen Nutzen der Forschung an Embryoiden nach 14 Tagen. Dazu gehören die Untersuchung der Ursachen von Unfruchtbarkeit, das Verständnis angeborener Geburtsfehler (Neuralrohr, Herz, Sinnesorgane usw.) und die Grundlagen von Entwicklungsstörungen. Wir kommen aus diesen Gründen zu dem Schluss, dass wir die Möglichkeit diskutieren sollten, vollständige Embryoide auch nach 14 Tagen zu kultivieren, und uns für ein erweitertes Regelwerk entscheiden sollten, das sowohl dem Menschen dient als auch anerkennt, dass kein menschliches Material - weder natürlich noch synthetisch ohne Prüfung und Aufsicht verwendet werden sollte.

\subsection{Empfehlungen für zukünftige Richtlinien}

Die Forschung an vollständigen synthetischen Modellen über 14 Tage hinaus sollte streng geregelt und an einer Reihe von Prinzipien orientiert sein. Sie könnte etwa kontextabhängig bewertet werden: Je weiter entwickelt Embryoide sind, desto mehr wissenschaftliche Begründungen, gesellschaftliche Vorteile und Kontrollen, Beurteilungen des Werts, der Absichten und Notwendigkeit sowie der Risiken und Nutzen des Forschungsprojekts sind erforderlich. Wir passen im Folgenden die ISSCR-Richtlinie 
aus dem Jahr 2016 an diese neuen Entitäten an und stützen uns dabei auf bisherige Empfehlungen von Wissenschaftler/-innen (Hyun et al., 2020):

(1) Wert: Verbesserungen der Gesundheit oder Erweiterungen des Wissens müssen aus der Forschung an vollständigen synthetischen Modellen über 14 Tage hinaus abgeleitet werden können. Um ethisch vertretbar zu sein, muss die Forschung an Embryoiden von Wert sein, insofern das wissenschaftliche Projekt eine Hypothese prüft, die wichtige Erkenntnisse über die menschliche Entwicklung hervorbringen kann. Das bedeutet nicht, dass die Forschung an Embryoiden unmittelbare praktische Auswirkungen haben muss. Grundlagenforschung ist für medizinische und klinische Innovationen unerlässlich. Das heißt, dass der Wert einer solchen Forschung von der Definition unserer sozialen und medizinischen Prioritäten abhängt und dass diese Forschung einen wichtigen Schritt hin zu diesen Prioritäten machen muss.

(2) Intention des Forschungsprojekts: „Die Absicht der Forschung sollte von den Regulierungsbehörden als das wichtigste ethische Kriterium angesehen werden“ (Rivron et al., 2018b: 185). Welche Verpflichtungen sind von der wissenschaftlichen Gemeinschaft eingegangen worden, um sich selbst Grenzen zu setzen? Als Teil einer Vereinbarung sollten menschliche Embryonenmodelle nicht zur direkten Verwendung in der assistierten Reproduktion mit dem Ziel der Erzeugung einer Schwangerschaft hergestellt werden. Sie könnten jedoch als In-vitro-Modelle verwendet werden, um die menschliche Fortpflanzung besser zu verstehen. Das Studium eines bestimmten definierten Entwicklungsabschnitts oder eines bestimmten anatomischen Strukturgefüges sollte nicht in dieselbe Kategorie fallen wie die Modellierung einer kontinuierlichen Entwicklung eines intakten Embryos oder Fetus. Diese Ansätze werfen nicht die gleichen ethischen Bedenken auf.

(3) Notwendigkeit: Das Forschungsprojekt sollte möglichst den Einsatz unnötiger Mittel vermeiden. Die Projektbeschreibung sollte eine Erörterung alternativer Methoden enthalten. Wenn es möglich ist, einen bestimmten definierten Entwicklungsabschnitt eines bestimmten anatomischen Strukturgefüges zu untersuchen, anstatt die kontinuierliche Entwicklung eines intakten Embryos oder Fetus zu modellieren, sollte dieser Ansatz bevorzugt werden. Bei Forschungsfragen, die ohne die Modellierung einer kontinuierlichen integrierten embryonalen Entwicklung über 14 Tage hinaus nicht vollständig beantwortet werden können, sollte die Forschung mit Kultursystemen, die die integrierte Entwicklung des Embryos nach der Gastrulation ermöglichen, obligatorisch überprüft werden.

4) Risiken/Nutzen: Bei Kultursystemen, die die integrierte Entwicklung des Embryos nach dem Auftreten des Primitivstreifens modellieren könnten, müssen wir eine öffentliche Debatte darüber eröffnen, 1) wie weit wir, je nach potenziellem Nutzen 
für die klinische und medizinische Forschung, bereit sind zu gehen und 2) wie wir den potenziellen Nutzen mit den potenziellen Risiken in ein Gleichgewicht bringen können. Wir haben oben das Fehlen eines Konsenses sowie der Gewissheit bezüglich des moralischen Status des Embryos und der Stadien, in denen er relevante moralische Merkmale aufweist, diskutiert. Aber wir müssen zumindest anerkennen, dass, je weiter das Entwicklungsstadium eines Embryoids fortgeschritten ist, es desto wahrscheinlicher ist, dass er einige relevante moralische Merkmale aufweist. Mit anderen Worten: Das Risiko, dass ein Embryoid in einem späteren Entwicklungsstadium einige relevante moralische Merkmale aufweist, muss Teil der Diskussion sein. Das Vorhandensein solcher moralischen Merkmale verbietet nicht notwendigerweise jede Art von Forschung, wie wir in unserem vorherigen Abschnitt über die Forschung an Menschen gezeigt haben. Ein solches Risiko (das in jedem Entwicklungsstadium unterschiedlich hoch ist) muss je nach der Bedeutung für und den Hinweisen auf einen künftigen potenziellen Nutzen abgeglichen werden. Abgesehen von den offensichtlichen Anwendungen auf Probleme der Unfruchtbarkeit sind auch die Ursachen von Entwicklungsstörungen noch unbekannt, und die Lösung von technischen Problemen wird höchstwahrscheinlich den Weg für die Herstellung von Organen für regenerative Therapien ebnen. Frühe angeborene oder sogar spät einsetzende Krankheiten entstehen oft in der frühen Embryogenese. Beispiele hierfür sind Autismus, Herzfehlbildungen und eine Vielzahl anderer Krankheiten, die ihre Wurzeln in der frühen Entwicklung haben. Autismus ist ein typisches Beispiel; falls wir je in der Lage sein sollten, die Ursache dessen zu verstehen, was bei autistischen Kindern abläuft, dann erfordert dies Kenntnisse darüber, was in den frühesten Stadien der neuronalen Entwicklung und der neuronalen Funktionen geschieht. Das früheste menschliche Gewebe, das für die Forschung zur Verfügung steht, stammt jedoch aus abgetriebenen menschlichen Feten, die in den meisten Fällen frühestens zwischen der 11. und 13. Woche nach der Empfängnis entnommen werden. Zwischen diesem Stadium und der vollständigen Einnistung des Embryos nach etwa 14 Tagen existiert daher eine Blackbox, die entscheidende Erkenntnisse über die Pathophysiologie der Entwicklungsstörung (Lehre von den Krankheitsvorgängen und Funktionsstörungen) enthält und für die Embryoide unschätzbare Informationen liefern könnten. In einem ersten Schritt schlagen wir daher vor, die Forschung sowohl an synthetischen als auch an natürlichen Embryonen auf bis zu 28 Tage auszudehnen, da das sensorische System in diesem Stadium noch zu unreif ist, um Empfindungsvermögen oder Schmerzen festzustellen (Hurlbut et al., 2017; Appleby/Bredenoord, 2018). Dies könnte der Startpunkt für die Erarbeitung der Rahmenbedingungen eines kontextabhängigen Umgangs sein, bei dem der Nutzen der Forschung gegenüber den Risiken von Fall zu Fall abgewogen wird. 
5) Transparenz und Beaufsichtigung: Die umfassende ISSCR-Richtlinie zeigt, wie wichtig ein strenges Monitoring durch eine speziell darauf ausgerichtete Beaufsichtigung der Forschung an menschlichen Embryonen (EMRO) ist. Diese Beaufsichtigung muss in Abhängigkeit von der Art der embryoähnlichen Entitäten (vollständig/partiell; vor oder nach 14 Tagen) erfolgen.

\subsection{Schlussfolgerungen}

Dieser Artikel ist ein Aufruf, eine pragmatische ethische Haltung in den Mittelpunkt der Debatte zu stellen. Wenn die Werte der verschiedenen Interessensgruppen in einer multikulturellen Gesellschaft nicht miteinander in Einklang gebracht werden können, müssen wir einen Rahmen finden, in dem wir ein umsichtiges Gespräch über diese neuen Technologien führen können. Obwohl beim gegenwärtigen Stand der Technik noch nicht nachgewiesen ist, dass sich diese Modelle länger als ein paar Tage in vitro entwickeln können, müssen wir davon ausgehen, dass die Zellkulturmethoden so weit verbessert werden könnten, dass die Modelle Schlüsselmerkmale der frühen menschlichen Entwicklung aufweisen und so die Unterschiede zum natürlichen Embryo minimieren. Wir drängen darauf, klar zwischen unseren individuellen Werten und unseren gesellschaftlichen Werten zu unterscheiden. Wir müssen mit dem Tempo der Wissenschaft Schritt halten und einen ethischen Rahmen entwerfen, der den Umgang mit diesen Entitäten trotz des Fehlens eines klaren Konsenses über den moralischen Status des intakten Embryos reguliert. Die oben vorgeschlagenen Empfehlungen sind als Beginn einer offenen öffentlichen Debatte zwischen den Wissenschaften, der Forschungspolitik, der Bioethik und der Öffentlichkeit zu verstehen.

\subsection{Literaturverzeichnis}

Aach, J. et al. (2017): Addressing the ethical issues raised by synthetic human entities with embryolike features. In: Elife, Online-Publikation 21.03.2017. DOI: 10.7554/eLife.20674.

Annas, G. J. et al. (1996): The politics of human-embryo research - Avoiding ethical gridlock. In: New England Journal of Medicine 335: 1243-1245.

Appleby, J. B./Bredenoord, A. L. (2018): Should the 14-day rule for embryo research become the 28-day rule? In: EMBO Mol. Med. 10(9): e9437.

Beccari, L. et al. (2018): Multi-axial self-organization properties of mouse embryonic stem cells into gastruloids. In: Nature 562(7726): 272-276.

Bok, S. (1988): Who shall count as a human being. In: Goodman, M. (Hrsg.): What is a Person? Humana Press, Totowa: 213-228. 
Chhabra, S. et al. (2019): Dissecting the dynamics of signaling events in the BMP, WNT, and NODAL cascade during self-organized fate patterning in human gastruloids. In: PLoS Biol. 17(10): e3000498.

Congregation for the Doctrine of the Faith (1987): Instruction on respect for human life in its origin and on the dignity of procreation. Replies to certain questions of the day. Unter: https://www.vati can.va/roman_curia/congregations/cfaith/documents/rc_con_cfaith_doc_19870222_respect-for -human-life_en.html [29.07.2020].

De Grazia, R. (2008): Moral status as a matter of degree? In: South. J. Philos. 46(2): 181-198.

Department of Health \& Social Security (1984): Report of the committee of inquiry into human fertilisation and embryology ('The Warnock Report'). Unter: https://www.hfea.gov.uk/media/260 8/warnock-report-of-the-committee-of-inquiry-into-human-fertilisation-and-embryology-1984.p df [29.07.2020].

Dong, C. et al. (2020): Derivation of trophoblast stem cells from naïve human pluripotent stem cells. In: Elife, Online-Publikation 12.02.2020. DOI: 10.7554/eLife.52504.

Douglas, T./Savulescu, J. (2009): Destroying unwanted embryos in research: Talking point on morality and human embryo research. In: EMBO Rep. 10: 307-312.

Ethics Advisory Board (1979): HEW support of research involving human in vitro fertilization and embryo transfer.

Unter: https://repository.library.georgetown.edu/bitstream/handle/10822/559350/HEW_IVF_rep ort.pdf?sequence=1\&isAllowed=y [29.07.2020].

Ford, N. M. (1989): When did I begin? Conception of the human individual in history, philosophy and science. Cambridge Univ. Press, Cambridge.

Gao, X. et al. (2019): Establishment of porcine and human expanded potential stem cells. In: Nat. Cell Biol. 21: 687-699.

George, R. P./Gómez-Lobo, A. (2005): The moral status of the human embryo. In: Perspect. Biol. Med. 48(2): 201-210.

Gleicher, N./Caplan, A. L. (2018): An alternative proposal to the destruction of abandoned human embryos. In: Nat. Biotechnol. 36(2): 139-141.

Gurdon, J. B. (1962): The developmental capacity of nuclei taken from intestinal epithelium cells of feeding tadpoles. In: J. Embryol. Exp. Morphol. 10: 622-640.

Gurmankin, A. D. et al. (2003) Embryo disposal practices in IVF clinics in the United States. In: Politics Life Sci. 22(2): 4-8.

Haremaki, T. et al. (2019): Self-organizing neuruloids model developmental aspects of Huntington's disease in the ectodermal compartment. In: Nat. Biotechnol. 37(10): 1198-1208.

Hurlbut, J. B. et al. (2017): Revisiting the Warnock rule. In: Nat. Biotechnol. 35(11): 1029-1042.

Hyun, I. et al. (2020): Toward guidelines for research on human embryo models formed from stem cells. In: Stem Cell Reports 14(2): 169-174. 
Iltis, A. et al. (2019): Human embryo research beyond day 14: Ethical questions and considerations. Unter: https://www.bakerinstitute.org/media/files/files/20b9e242/chb-pub-greenwall-ethics-021 219.pdf [29.07.2020].

ISSCR (2016): Guidelines for stem cell science and clinical translation. Unter: https://www.isscr.org/ docs/default-source/all-isscr-guidelines/guidelines-2016/isscr-guidelines-for-stem-cell-research -and-clinical-translationd67119731dff6ddbb37cff0000940c19.pdf?sfvrsn=4 [29.07.2020].

Li, R. et al. (2019): Generation of blastocyst-like structures from mouse embryonic and adult cell cultures. In: Cell 179(3): 687-702.

Matthews, K./Yang, E. A (2018): Drawing the line: Assessing U.S. human embryo research and the 14-day limit. Unter: https://www.bakerinstitute.org/media/files/files/a9096889/chb-pub-greenw all-hesc-011519.pdf [29.07.2020].

Moris, N. et al. (2020): An in vitro model of early anteroposterior organization during human development. In: Nature 582(7812): 410-415.

Rivron, N. C. et al. (2018a): Blastocyst-like structures generated solely from stem cells. In: Nature 557(7703): 106-111.

Rivron, N. C. et al. (2018b): Debate ethics of embryo models from stem cells. In: Nature 564(7735): 183-185.

Sandel, M. J. (2005): The ethical implications of human cloning. In: Perspect. Biol. Med. 48(2): 241247.

Shahbazi, M. N. et al. (2019): Self-organization of stem cells into embryos: A window on early mammalian development. In: Science 364(6444): 948-951.

Shao, Y. et al. (2017): A pluripotent stem cell-based model for post-implantation human amniotic sac development. In: Nat. Commun. 8(1): 208.

Simunovic, M. et al. (2018): Molecular mechanism of symmetry breaking in a 3D model of a human epiblast. In: bioRxiv, Online-Publikation 29.05.2018. DOI: 10.1101/330704.

Singer, P./Kuhse, H. (1986): Debate: Embryo research: The ethics of embryo research. In: J. Law, Med. Ethics 14(3-4): 133-138.

Sozen, B. et al. (2018): Self-assembly of embryonic and two extra-embryonic stem cell types into gastrulating embryo-like structures. In: Nat. Cell Biol. 20(8): 979-989.

Sozen, B. et al. (2019): Self-organization of mouse stem cells into an extended potential blastoid. In: Dev. Cell 51(6): 698-712.e8.

Warmflash, A. et al. (2014): A method to recapitulate early embryonic spatial patterning in human embryonic stem cells. In: Nat. Methods 11(8): 847-854. 